\title{
CONGENITAL HEART DISEASE
}

\section{Low pressure giant pulmonary artery aneurysms in the adult: natural history and management strategies}

\author{
G R Veldtman, J A Dearani, C A Warnes
}

Heart 2003;89:1067-1070

See end of article for authors' affiliations

Correspondence to:

Dr C A W arnes, Mayo

Medical School, Mayo

Clinic, 200 First Street SW

Rochester, Minnesota

55905, USA:

warnes.carole@mayo.edu

Accepted 7 April 2003
Objective: To describe aspects of the natural history and pathophysiology of giant low pressure pulmonary artery aneurysms and to propose potential surgical strategies.

Design: Cross sectional retrospective review.

Setting: Supraregional tertiary referral centre.

Patients: All adult patients referred for assessment of giant pulmonary artery a neurysm retrospectively identified from the Mayo Adult Congenital Heart Disease Clinic database.

Methods: Patient data were reviewed from hospital records, including echocardiograms, magnetic resonance images, radiographs, and histology slides.

Results: Four patients were identified with a median age of 52 years (range 37-64 years). Presenting symptoms were effort related dyspnoea, chest discomfort, and hoarseness in one patient. All patients had pulmonary regurgitation and clinical evidence of right ventricular enlargement in association with a pulsatile mass at the upper left sternal edge. Transthoracic echocardiography showed the giant pulmonary artery aneurysm involving the main pulmonary artery and proximal branches, and confirmed severe pulmonary regurgitation in all patients. $\mathrm{N}$ one of the patients had intimal tearing, medial dissection, or pulmonary arterial rupture. The pulmonary valve was replaced to relieve symptoms and preserve right ventricular function. Pulmonary arterial histology showed medial degeneration of elastic fibres with accumulation of basophilic ground substance.

Conclusions: Rupture or dissection of these low pressure aneurysms is rare. The timing of surgical intervention should be determined by changes in right ventricular size and function resulting from pulmonary regurgitation or pulmonary stenosis, and not the size of the aneurysm.
S poradically patients may present with giant pulmonary artery aneurysms-that is, with diameters in excess of $5 \mathrm{~cm}$. Under these circumstances, it may be presumed that intrinsic arterial wall weakness is inherent and progression is inevitable, and that complications of dissection and rupture are part of the natural history. In those patients with pulmonary artery aneurysm and significant primary or secondary pulmonary hypertension caused by a left to right shunt, this is undoubtedly the case. ${ }^{1} \mathrm{H}$ owever, the natural history of low pressure giant pulmonary artery aneurysms is not yet defined and management is often controversial. We present four adult patients with giant low pressure pulmonary artery aneurysms, document features of the natural history and pathophysiology, and propose potential surgical strategies.

\section{CASE 1}

A 59 year old woman reported progressive exertional dyspnoea over 15 years and recent hoarseness. She had had an asymptomatic heart murmur during childhood. She had normal jugular venous pressures, a prominent right ventricular impulse, and a large area of pulsatility in the pulmonary area on examination. Auscultation showed a pulmonary ejection click followed by a short ejection systolic murmur, a wide split second sound, and a long diastolic murmur of pulmonary regurgitation. She had no stigmata of a connective tissue disorder. Figure 1 shows the chest radiograph.

Transthoracic echocardiography confirmed severe pulmonary regurgitation, severe right ventricular enlargement with preserved systolic function, a tiny persistent ductus arteriosus, and a $9 \mathrm{~cm}$ aneurysm involving the entire main pulmonary artery and the proximal branches. Because of the severe pulmonary regurgitation and severe right ventricular enlargement, she underwent surgical repair. The pulmonary valve, which had one hypoplastic cusp, was excised along with the main pulmonary artery and replaced with a $26 \mathrm{~mm}$ Hancock valved conduit extending to the bifurcation. The small ductus

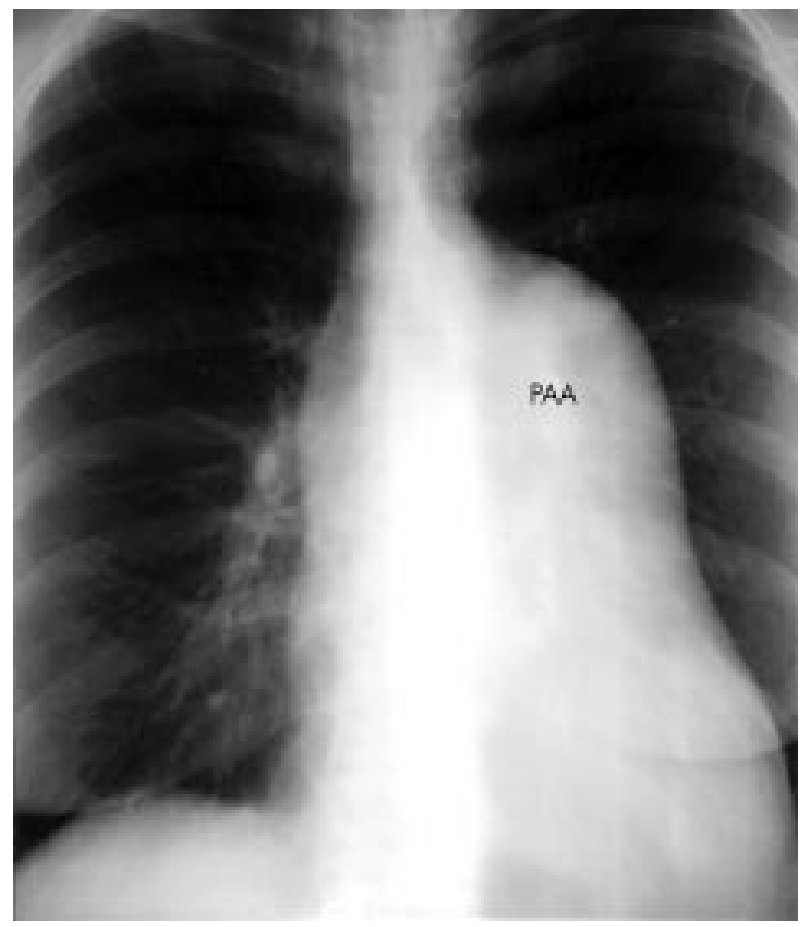

Figure 1 Case 1: chest radiograph (posteroanterior projection). PAA, pulmonary artery a neurysm. 


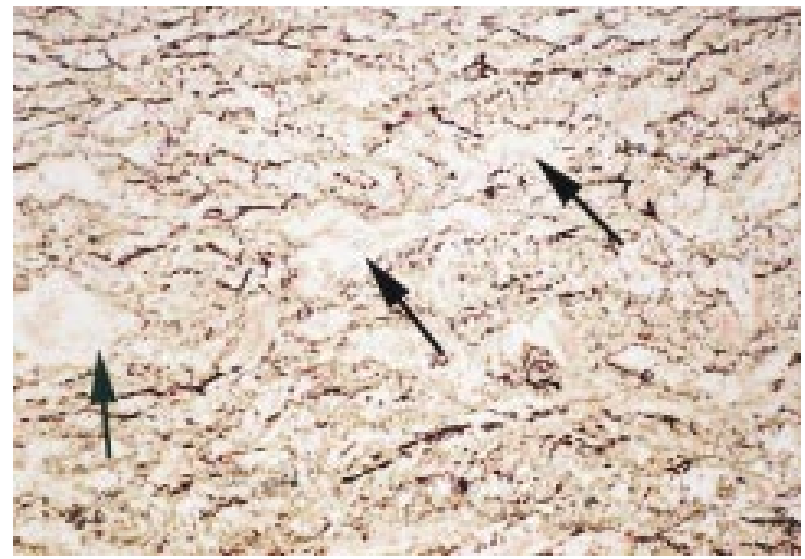

Figure 2 Case 1: histology of aneurysmal pulmonary arterial wall. Elastic van $\mathrm{G}$ ieson stain. Notice fragmentation and associated loss of the elastic fibres with increased ground substance in these areas, indicated by black arrows.

arteriosus was closed. In the pulmonary artery there was no intimal tear, medial dissection, or calcification. Histology showed medial degeneration and fragmentation of the elastic fibres with increased ground substance but no loss of smooth muscle cells or inflammation ( fig 2). At six months' follow up, her symptoms had improved significantly and her right ventricle was only moderately dilated with a pressure of $46 \mathrm{~mm} \mathrm{Hg}$.

\section{CASE 2}

A 37 year old man with dextrocardia and longstanding pulmonary regurgitation reported progressive dyspnoea for the preceding decade. Cardiac catheterisation had been performed at 10 days of age confirming pulmonary stenosis and regurgitation, but he was asymptomatic until young adulthood. On examination, he had no features of a

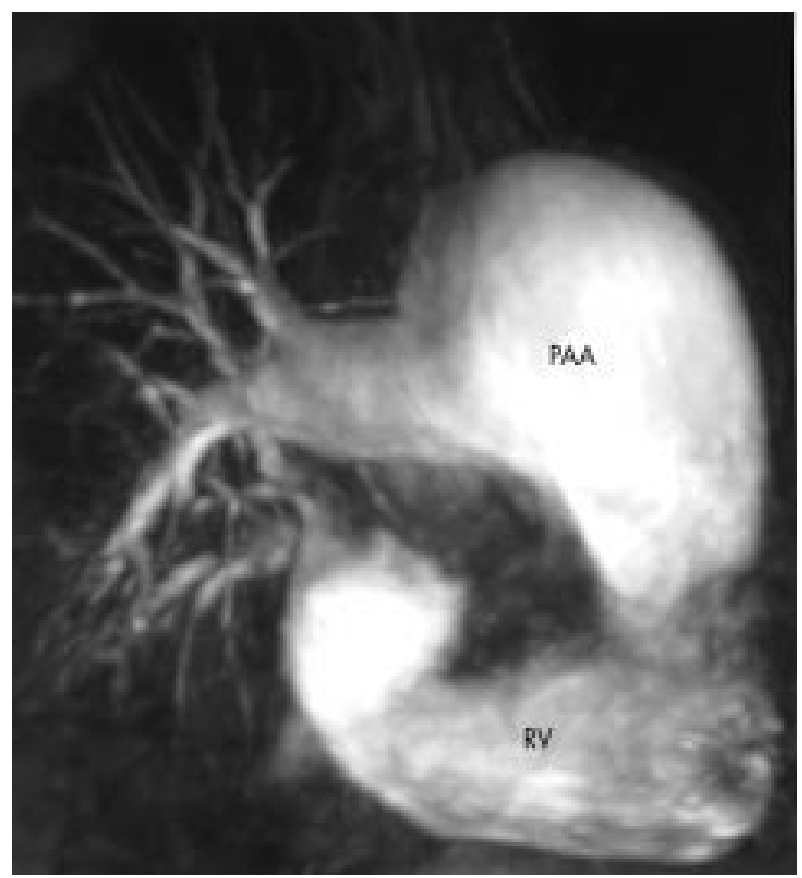

Figure 3 Case 2: three dimensional reconstruction of magnetic resonance angiography with gadolinium enhancement. The a neurysm extends to the branch pulmonary arteries. PAA, pulmonary artery a neurysm; RV, right ventricle.

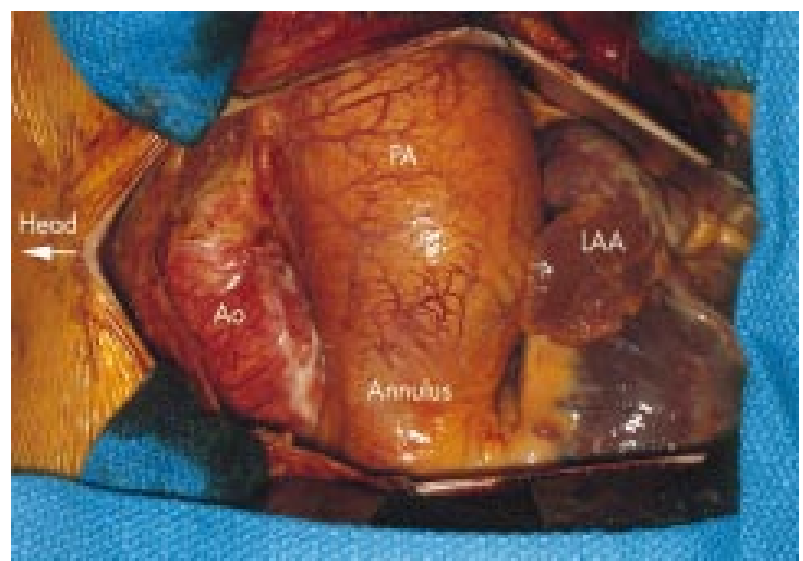

Figure 4 Case 2: surgical view of the pulmonary artery aneurysm (PA). A o, ascending aorta; LAA, left atrial appendage.

connective tissue disorder but had a pectus excavatum. J ugular venous pressure was normal. He had an ejection click with a short ejection systolic murmur, a normal second sound, and a soft murmur of pulmonary regurgitation. Transthoracic echocardiography, intraoperative transoesophageal echocardiogram, and magnetic resonance imaging (fig 3) confirmed an $8 \mathrm{~cm}$ pulmonary artery aneurysm with severe pulmonary regurgitation and moderate right ventricular dilatation with mild systolic dysfunction. There was also mild ascending aortic dilatation to $43 \mathrm{~mm}$ but no aortic regurgitation. At operation (fig 4), his bicuspid pulmonary valve was replaced with a $31 \mathrm{~mm}$ Carpentier-Edwards bioprosthesis and he had aneurysmorrhaphy of the main pulmonary artery extending to, but not including, the branches. Histology showed medial degeneration and fragmentation of the elastic fibres and accumulation of ground substance.

\section{CASE 3}

A 64 year old woman was evaluated for a three year history of dyspnoea, pulmonary regurgitation, and pulmonary artery aneurysm. At age 13 years, she had a Brock procedure for congenital valvar pulmonary stenosis. She had been a longstanding smoker and had both peripheral vascular disease and obstructive airways disease. On examination, she had features of pulmonary stenosis and pulmonary regurgitation. Her jugular venous pressure was increased to $10 \mathrm{~cm} \mathrm{H}_{2} \mathrm{O}$ with prominent a-waves. She had a notable right ventricular lift extending into a pulsatile mass at the upper left sternal border. On two dimensional echocardiography, the main pulmonary artery was dilated to $7 \mathrm{~cm}$, pulmonary regurgitation was severe, and right ventricular outflow gradient measured $54 \mathrm{~mm} \mathrm{Hg}$ (mean $18 \mathrm{~mm} \mathrm{Hg}$ ). Despite severe dilatation, the right ventricle had only mildly reduced systolic function and mild tricuspid regurgitation. Right ventricular systolic pressure was $75 \mathrm{~mm} \mathrm{Hg}$. At operation, the pulmonary valve had three cusps with annular dilatation and there was no evidence of intimal tear or medial dissection of the pulmonary artery. The pulmonary valve was replaced with a $29 \mathrm{~mm}$ CarpentierEdwards porcine bioprosthesis. The main pulmonary artery was plicated. Histology showed a thinned media with focal loss of elastic fibres. Postoperatively the right ventricle was only mildly enlarged with normal function.

\section{CASE 4}

A 45 year old hypertensive man with non-insulin dependent diabetes presented with two years of effort related dyspnoea. An asymptomatic pulmonary murmur was noted at age 15 and chest radiography showed a pulmonary artery aneurysm. He had had no follow up. He had no stigmata of connective tissue disease. He had normal jugular venous pressures, a right 
ventricular lift, and murmurs of pulmonary regurgitation and increased pulmonary flow. Chest radiograph showed a massive main pulmonary artery measuring $10 \mathrm{~cm}$ on echocardiography. The pulmonary valve, which was calcified but not stenotic, had severe regurgitation with associated moderate right ventricular dilatation. A coronary angiogram showed areas of severe fusiform dilatation of the epicardial coronary arteries without significant stenosis. At operation, yellow atheromatous plaques were visible in the multiple coronary aneurysms, which were easily seen. The trileaflet pulmonary val ve had two dysplastic cusps and was replaced with a $29 \mathrm{~mm}$ Carpentier-Edwards porcine bioprosthesis. Aneurysmorrhaphy of the main and proximal branch pulmonary arteries was also performed. There was no evidence of intimal tear or medial dissection. Histology showed severe medial degeneration. Histology of the ascending aorta was normal. At latest follow up 24 months after surgery, his symptoms had resolved. Right ventricular function and size had returned to normal. The patient remains on warfarin for the coronary artery aneurysms.

\section{DISCUSSION}

True pulmonary artery aneurysms are rare. Deterling and Clagett reported only eight cases in a review of 109571 necropsies. ${ }^{2}$ Aggressive surgical approaches have often been advocated based on previous reports of dissection or rupture. Pulmonary artery aneurysms, even when very large, in the presence of low pulmonary pressures and the absence of left to right congenital or acquired shunts, may not pose such a risk in sharp contrast with those with pulmonary hypertension or Eisenmenger's syndrome. ${ }^{34}$ Patients in this series all had low pulmonary arterial pressures, no significant congenital or acquired left to right shunts, and giant pulmonary artery aneurysms involving the main or proximal branch pulmonary arteries. They survived to a median age of 52 years without evidence of intimal tear or medial dissection and presented because of right ventricular dysfunction secondary to pulmonary regurgitation.

True aneurysms involve all components of the vessel wall. ${ }^{5}$ There may be associated intrinsic weakness of the arterial wall compounded by the presence of increased haemodynamic shear stresses. It is therefore not surprising that pulmonary arterial aneurysm has been described in patients with persistent ductus arteriosus, ventricular septal defect, atrial septal defect, and transposition of the great arteries with ventricular septal defect, where the pulmonary circulation may have considerable volume and pressure overload. ${ }^{26} \mathrm{M}$ ore recently, associated connective tissue disorders such as Marfan's or the Ehlers-Danlos syndrome have been identified and systemic vasculitides to a lesser extent. Case 4 involved an unusual combination of coronary artery, ascending aorta, and pulmonary arterial dilatation. This patient, however, had no features to suggest connective tissue disorder and histology excluded vasculitis.

All four of our patients had structurally abnormal pulmonary valves on surgical inspection. In case 1 , a single cusp was hypoplastic. One patient (case 2) had a bicuspid pulmonary valve. One patient (case 3 ) had a dysplastic tricuspid valve with mild valvar stenosis and one (case 4) had two thickened valve cusps. It is reasonable to presume that these valves were congenitally malformed. Additionally, all four patients had degeneration and fragmentation of elastic fibres in the pulmonary artery media and accumulation of ground substance, findings consistent with so called "cystic medial necrosis". These findings are similar to those in patients with aortic root dilatation in the presence of a dysplastic bicuspid aortic valve, ${ }^{7}$ in whom it has been postulated that a developmental abnormality may give rise to the intrinsic arterial wall weakness as described above. ${ }^{8}$ Haemodynamic perturbation from the abnormally opening valve, even in the absence of stenosis, may induce apoptosis, remodelling, and aneurysmal transformation of the vessel wall. ${ }^{8} \mathrm{In}$ the presence of regurgitation, the haemodynamic stress may be exacerbated, as stroke volume is increased, resulting in the largest root aneurysms. ${ }^{9}$ It may be reasonable to extrapolate these mechanisms to the right side of the heart.

The natural history of low pressure pulmonary arterial aneurysms was hitherto unknown. Deterling, Senbaklavaci, and others have documented progression to dissection or rupture in the setting of severe pulmonary arterial hypertension, and patients often had associated congenital left to right shunts. ${ }^{12}$ Similarly, evidence of progression to arterial wall dissection and rupture has been documented in patients with connective tissue disorders. ${ }^{10}$ Our four patients all survived well into adulthood despite aneurysms ranging in size from 7-10 cm without dissection. The law of Laplace dictates that wall stress, the most important determinant of progression to rupture, is directly proportional to the pressure and radius of the vessel wall and is inversely proportional to the wall thickness. ${ }^{11}$ In our patients, pulmonary artery pressures were low or only mildly increased (case 2), implying relatively lower wall stress, despite the massively increased vessel diameter.

Our patients all had congenitally malformed valves and complications related to longstanding pulmonary regurgitation, including right ventricular enlargement and dysfunction and effort related dyspnoea. One patient (case 1) also had hoarseness. This rare complication is usually seen in aortic root dilatation or severe mitral stenosis (Ortner's syndrome)..$^{12}$

The management of pulmonary artery aneurysm has largely been surgical. Reported procedures have included Dacron interposition graft placement, autologous pericardial replacement of the main pulmonary artery, and pulmonary arterial aneurysmorrhaphy. ${ }^{13-15}$ One of our patients had a Dacron graft replacement and the other patients had pulmonary arterial aneurysmorrhaphy/plication. All had pulmonary valve replacement, thereby relieving not only right ventricular volume overload but also the associated haemodynamic burden on the vessel wall as described earlier. In the surgical series of Kuwaki and colleagues, ${ }^{15}$ aneurysmorrhaphy, without pulmonary valve replacement, was associated with late recurrence of pulmonary arterial dilatation, perhaps because of the persistent haemodynamic stress and defective native pulmonary arterial wall.

In the absence of significant pulmonary regurgitation or stenosis (that is, enough to cause right ventricular dysfunction), pulmonary hypertension, or associated left to right shunt, a conservative strategy appears to be deductive. The risk of aneurysm rupture seems very small and not surprisingly various authors have confirmed late survival with conservative management. ${ }^{316}$ Patients are more likely to present with complications of pulmonary regurgitation.

In conclusion, we believe that the above cases shed light on important aspects of the pathophysiology and natural history of patients with low pressure giant pulmonary arterial aneurysms. These aneurysms are probably the result of a combination of intrinsic arterial wall weakness and haemodynamic stress, especially when congenital malformation of the valve leads to regurgitation. Stenosis does not need to be present to induce vessel wall remodelling and dilatation. The natural history includes right ventricular dysfunction secondary to volume overload, which prompted intervention in our series. Concomitant repair of the aneurysm may not be necessary but appears to be a strategically sound approach when the patient is already having open heart surgery for severe pulmonary regurgitation.

\section{ACKNOWLEDGEMENTS}

The authors acknowledge Drs GK Danielson, HV Schaff, and K Chandrasekaran, who generously provided clinical information on the patients. 
Authors' affiliations

G R Veldtman, C A Warnes, Division of Cardiovascular Diseases and Internal M edicine, Mayo Clinic, Rochester, Minnesota, USA

J A Dearani, Division of Cardiovascular Surgery, Mayo Clinic

\section{REFERENCES}

1 Senbaklavaci $O$, Kaneko Y, Bartunek A, et al. Rupture and dissection in pulmonary artery aneurysms: incidence, cause, and treatment: review and case report. J Thorac Cardiovasc Surg 2001;121:1006-8.

2 Deterling RA, Clagett T. A neurysm of the pulmonary artery: review of the literature and report of a case. Am Heart J 1947;34:471-99.

3 Casselman $F$, M eyns $B$, Herygers $P$, et al. Pulmonary artery aneurysm: is surgery always indicated? Acta Cardiol 1997;52:431-6.

4 Daliento L, Somerville J, Presbitero P, et al. Eisenmenger syndrome: factors relating to deterioration and death. Eur Heart 1998; 19:1845-55

5 Keene RJ, Steiner RE, O Isen EJ, et al. A ortic root aneurysm: radiographic and pathologic features. Clin Radiol 1971;22:330-40.

6 Ansari A. Isolated pulmonary valvular regurgitation: current perspectives. Prog Cardiovasc Dis 1991;33:329-44.
7 McKusick VA Association of congenital bicuspid aortic valve and Erdheim's cystic medial necrosis. Lancet 1972;i:1026-7.

8 Bonderman D, G harehbaghi-Schnell E, W ollenek G, et al. Mechanisms underlying aortic dilatation in congenital aortic valve malformation. Circulation 1999;99:2138-43.

9 Hahn RT, Roman MJ, Mogtader AH, et al. Association of a ortic dilation with regurgitant, stenotic and functionally normal bicuspid aortic valves. J Am Coll Cardiol 1992;19:283-8.

10 Westaby S. M anagement of a ortic dissection. Curr O pin Cardiol $1995 ; 10: 505-10$

11 11. Butto F, Lucas RV Jr, Edwards JE. Pulmonary arterial a neurysm: a pathologic study of five cases. Chest 1987;91:237-41.

12 Sengupta A, Dubey SP, Chaudhuri D, et al. O rtner's syndrome revisited. J Laryngol O tol 1998;112:377-9.

13 Arom KV, Richardson JD, G rover FL, et al. Pulmonary artery a neurysm. Am Surg 1978;44:688-92.

14 Casselman F, Deferm H, Peeters $P$, et al. A neurysm of the left pulmonary artery: surgical allograft repair. Ann Thorac Surg 1995;60:1423-5.

15 Kuwaki K, M orishita K, Sato H, et al. Surgical repair of the pulmonary trunk a neurysm. Eur J Cardiothorac Surg 2000;18:535-9.

16 van Rens MT, W estermann CJ, Postmus PE, et al. Untreated idiopathic aneurysm of the pulmonary artery; long-term follow - up. Respir Med $2000 ; 94: 404-5$

\section{IMAGES IN CARDIO LO G Y}

\section{Suspected bilateral renal artery stenosis}

A 49 year old man was referred to our interventionist for percutaneous revascularisation for suspected bilateral renal artery stenosis. Cardiovascular risk factors included smoking, hypercholesterolaemia, and recently diagnosed hypertension. Renal function was normal. Doppler examination of the renal arteries was arranged because of poorly controlled hypertension. Doppler waveform of both renal arteries showed "tardus parvus" pattern (below, R.RA, right renal artery; L.RA, left renal artery) suggestive of significant bilateral renal arteries stenosis.

Physical examination before cardiac catheterisation, however, revealed weak bilateral lower limb pulses, radio-femoral delay and ejection systolic murmur over the back.

Cardiac catheterisation via the femoral approach documented coarctation of aorta with associated $100 \mathrm{~mm} \mathrm{Hg}$ pressure gradient (right, upper panel). There was associated post-stenotic dilatation. Bilateral renal arteries were normal.

Subsequent magnetic resonance arteriography confirmed a segmental coarctation of the thoracic aorta immediately distal to the takeoff of the left subclavian artery (right, lower panel, arrow points to coarctation). Subsequently, the patient underwent surgical repair and had an uneventful recovery.

P-Y Lee

W Ng

W-H Chen

lee.pui-yin@graduate.hku.hk
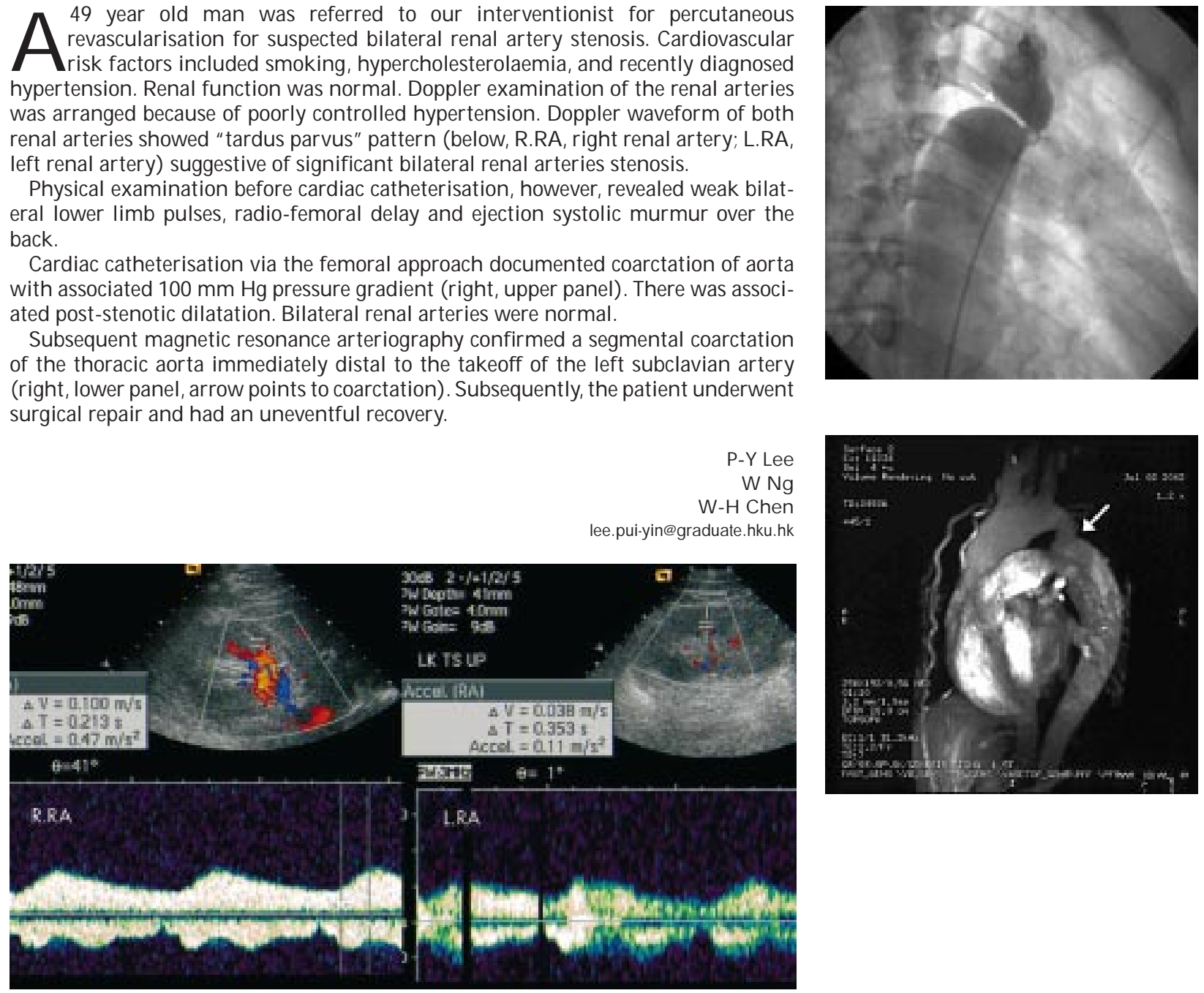